\title{
Miocardiopatía inducida por arritmia: informe de caso
}

\author{
Arrhythmia-induced cardiomyopathy: case report
}

Ana C. Gonzales-Luna ${ }^{1,2 *}$, Pedro Segura-Saldaña ${ }^{1,3,4}$, Rodrigo León-Vivar ${ }^{1,5}$, Jonny Laurente-Gómez ${ }^{1}$ y
Patricia Ríos-Navarro 1,5
${ }^{1}$ Departamento de Cardiología, Hospital Nacional Edgardo Rebagliati Martins; ${ }^{2}$ Clínica Delgado; ${ }^{3}$ Facultad de Ingeniería Biomédica, Universidad
Peruana Cayetano Heredia; ${ }^{4}$ Department of Cardiology Research, Torres de Salud National Research Center; ${ }^{5}$ Clínica Ricardo Palma. Lima, Perú

La miocardiopatía inducida por arritmia (MClA) es una alteración potencialmente reversible, en la cual la disfunción ventricular es inducida o mediada por arritmias auriculares o ventriculares. El reconocimiento temprano de la MCIA y el tratamiento temprano de la arritmia causal, mediante fármacos o técnicas de ablación, tienen como resultado la resolución de los síntomas y la recuperación de la función ventricular ${ }^{1}$.

\section{Caso clínico}

Se presenta el caso de un varón de 41 años, sin comorbilidad previa conocida que ingresó a emergencias por disnea progresiva y ortopnea de dos semanas de evolución. A su ingreso se lo encontró normotenso y taquicárdico, con signos de insuficiencia cardíaca congestiva y ruidos cardíacos irregulares. El electrocardiograma (ECG) (Fig. 1A) documentó flúter auricular con respuesta ventricular alta. Recibió amiodarona y lanatósido $\mathrm{C}$ intravenosos, pero cursó con prolongación del intervalo QT (QTc, 550 m) y múltiples episodios de fibrilación ventricular y taquicardia helicoidal (torsades de pointes) que requirieron desfibrilación.

El ecocardiograma reveló miocardiopatía dilatada (ventrículo izquierdo [VI] en diástole $[65 \mathrm{~mm}$ ] y aurícula izquierda [50 mm] de diámetro), con disfunción sistólica grave (fracción de expulsión del VI de 28\%) e hipocinesia global. El cateterismo cardíaco no mostró lesiones coronarias. El estudio electrofisiológico confirmó flúter auricular del istmo cavotricuspídeo dependiente (Fig. 1ByC), sometido a ablación y su bloqueo bidireccional. El paciente evolucionó de manera favorable y recibió el alta con bisoprolol, enalaprilo y warfarina. En el seguimiento, el ecocardiograma a los cuatro meses mostró reversión del diámetro de cavidades y mejoría de la función sistólica del VI (62\%) (Fig. 2). $\mathrm{Al}$ año de evolución se ha mantenido el ritmo sinusal.

\section{Discusión}

La MCIA se describió por primera vez en 1913 y su naturaleza reversible se notificó en $1962^{1}$. La MCIA se define como la disfunción sistólica del VI (DSVI) secundaria a una taquiarritmia patológica (paroxística o persistente) o una ectopia muy frecuente, auricular o ventricular $^{1,2}$. La característica principal de la MCIA es la recuperación de la función del VI, ya sea completa o parcial, después de la supresión o control de la arritmia.

La incidencia de la MCIA es también variable según sea el tipo de arritmias; se han publicado incidencias de $8 \%$ a $36 \%$ en taquicardias auriculares ectópicas focales y $9 \%$ a $34 \%$ en la ectopia ventricular y la taquicardia ventricular no sostenida. En el caso de la taquicardia auricular ectópica (TAE), Velásquez, et al. informaron un $36 \%$ con TAE resistente y $\mathrm{MCIA}^{3}$. En el flúter

\section{Correspondencia:}

*Ana C. Gonzales-Luna

E-mail: anace18@hotmail.com
Disponible en internet: 02-07-2021 Arch Cardiol Mex. 2021;91(3):379-382 www.archivoscardiologia.com

1405-9940 / @ 2021 Instituto Nacional de Cardiología Ignacio Chávez. Publicado por Permanyer. Este es un artículo open access bajo la licencia CC BY-NC-ND (http://creativecommons.org/licenses/by-nc-nd/4.0/). 


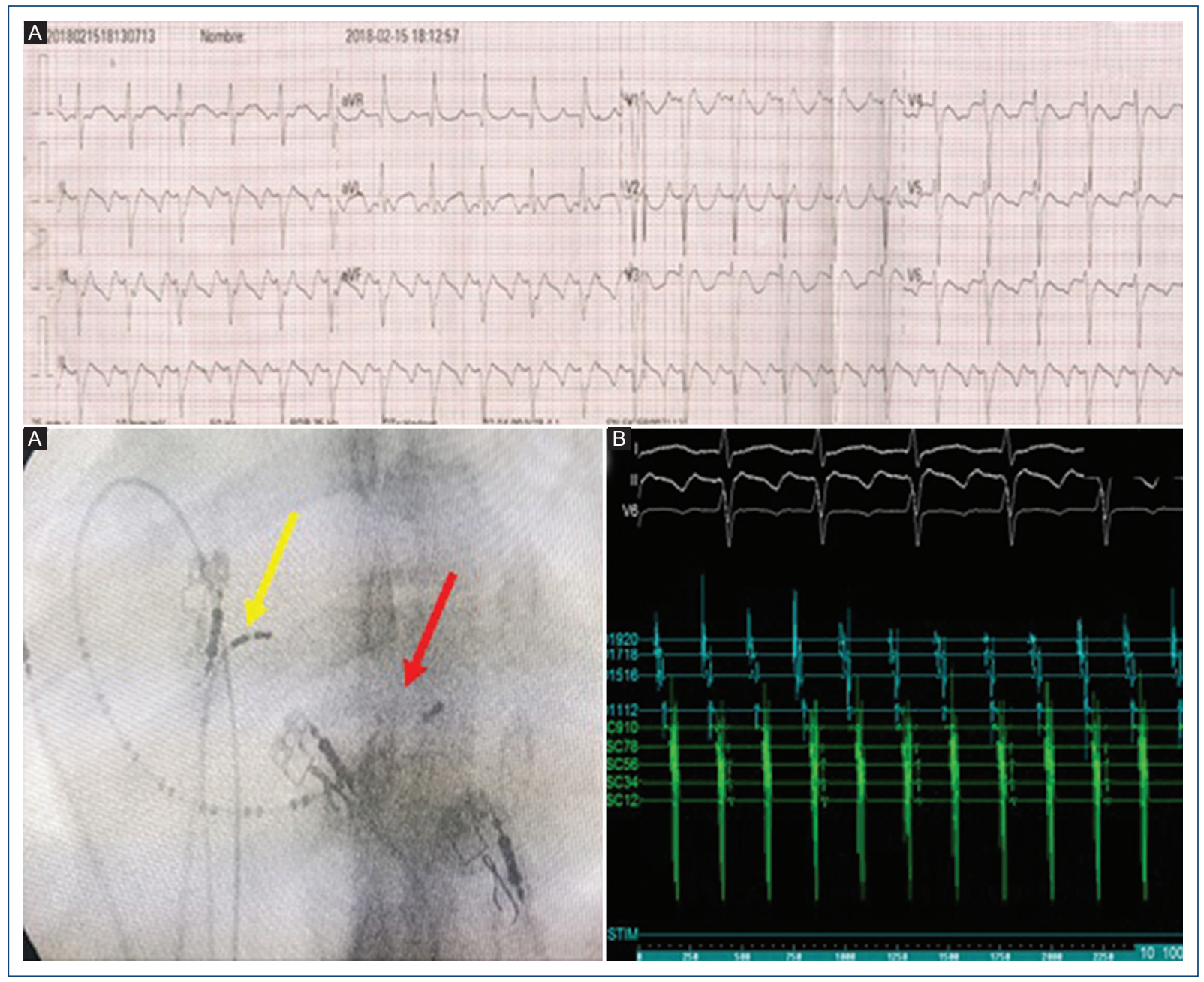

Figura 1. Ecocardiografía: imágenes en el eje paraesternal largo. A: Estudio ecocardiográfico al ingreso del paciente; se observa dilatación del VI (diámetro de fin de la diástole y sístole: $65 \mathrm{~mm}$ y $58 \mathrm{~mm}$, respectivamente). B: Ecocardiograma de control luego de cuatro meses de la ablación; se identifica la reducción de ambos diámetros (diámetro de fin de la diástole: $52 \mathrm{~mm}$; fin de la sístole: $35 \mathrm{~mm}$ ), con mejoría de la función sistólica del VI.

auricular se ha descrito hasta en un $25 \%$. En un estudio de 1,269 pacientes con flúter auricular se encontró una incidencia de MCIA del $8 \%$ y frecuencias ventriculares más altas en comparación con la DSVI de otra causa $(109 \pm 19 \text { vs. } 84 \pm 23 / \text { min, respectivamente })^{4}$. Se ha señalado que la frecuencia cardíaca a partir de la cual se produce la MCIA es $>100$ latidos por minuto en forma persistente.

Se han propuesto dos tipos de MCIA:

- Tipo 1: inducida por arritmia. La miocardiopatía (MC) ocurre de novo en un corazón estructuralmente normal y la arritmia es la única causa. La función del VI se normaliza después del tratamiento de la arritmia ${ }^{2}$.

- Tipo 2: mediada por arritmia. La arritmia exacerba la cardiopatía estructural preexistente y el tratamiento de la arritmia tiene como resultado una resolución parcial de la $\mathrm{MC}^{2}$.

La presentación clínica es variable, desde la asintomática (10\%) hasta la insuficiencia cardíaca (IC) terminal. Los síntomas más informados son palpitaciones $(29 \%)$, síntomas de insuficiencia cardíaca $(47 \%)$ y síncope o presíncope $(12 \%)^{5}$. La muerte súbita es infrecuente, pero se ha comunicado hasta en $8 \%$ a $12 \%$ a pesar del tratamiento y la resolución de la $\mathrm{MCIA}^{6}$.

Los síntomas se manifiestan en fase más temprana con FC más altas, como en el flúter auricular persistente o las taquicardias con conducción 2:16.

La clave diagnóstica es la detección de una arritmia en presencia de DSVI inexplicable. Un sólo ECG de 12 


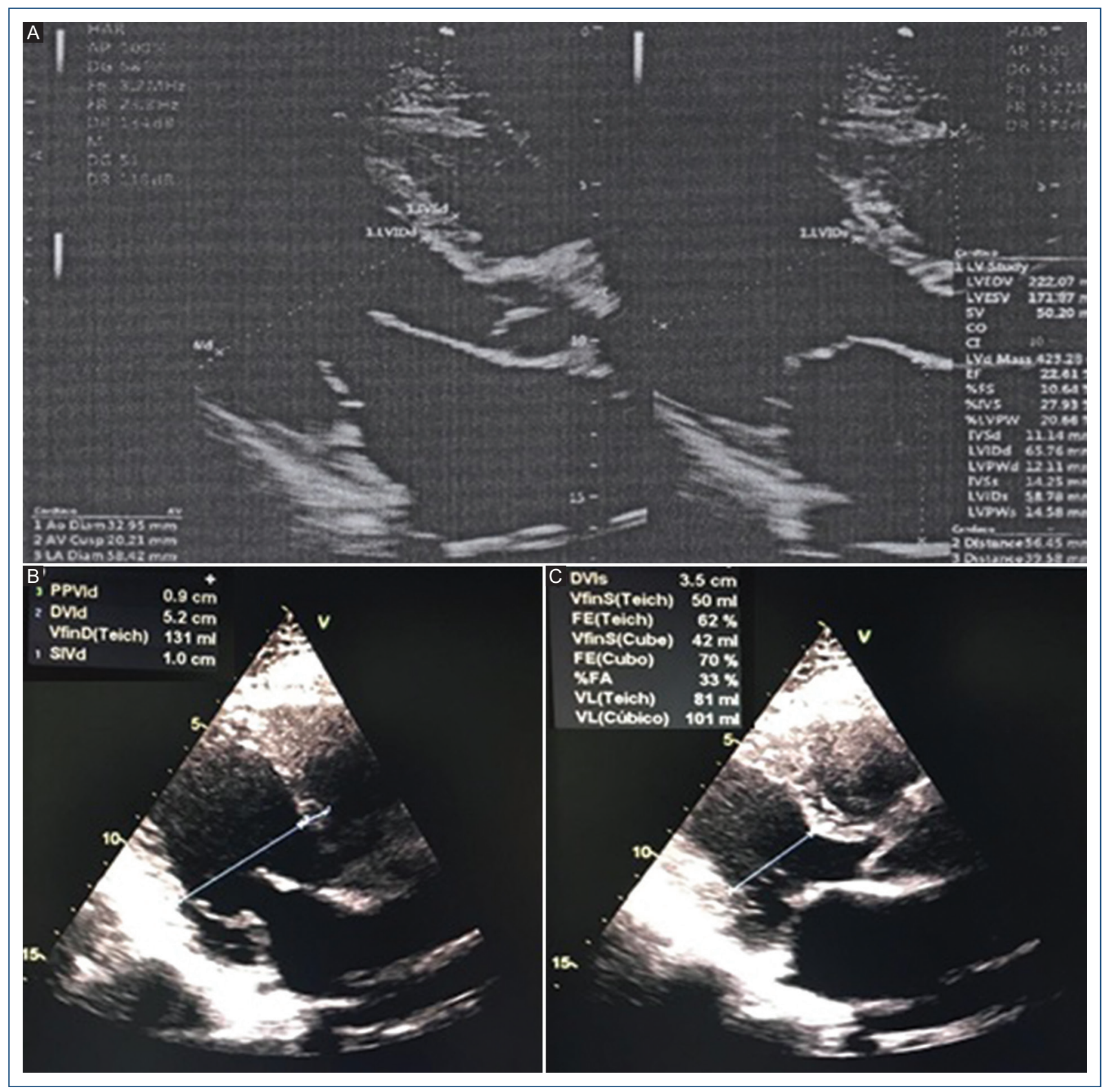

Figura 2. A: El ECG delinea flúter auricular con conducción 2:1, FC de 136 Ipm y ondas F negativas en DII, DIII, aVF y positivas en V1. B: Radiografía de tórax en proyección oblicua anterior izquierda que muestra un catéter duodecapolar en anillos mitral y tricuspídeo (flecha roja) y catéter cuadripolar a nivel del His (flecha amarilla). C: Registro intracavitario del flúter auricular en el estudio electrofisiológico. Los tres primeros canales son evidencia del electrocardiograma de superficie (derivaciones DI, DIl y V6) y los restantes evidencia de los electrocardiogramas intracavitarios documentados por el catéter duodecapolar: los cinco canales inferiores (color verde SC1-2 a SC 9-10) registran la señal a nivel del seno coronario (anillo mitral) y los canales superiores (color celeste SC11-12 a SC 10 -20) registran el catéter en la aurícula derecha (anillo tricuspídeo). Se observa una actividad continua en estos electrocardiogramas intracavitarios durante el flúter auricular.

derivadas puede no diagnosticar la arritmia causal; la vigilancia ambulatoria con Holter ayuda a identificar la taquicardia recurrente, la frecuencia ventricular media en fibrilación o flúter auricular y la frecuencia de complejos ventriculares prematuros ${ }^{1}$. Los pacientes con MCIA tienen un diámetro diastólico final del VI e índice de masa del VI menores, en comparación con aquéllos con $\mathrm{CM}$ dilatada preexistente. 
Los marcadores neurohormonales están elevados, de acuerdo con el grado de IC, y su descenso súbito en la primera semana de eliminada o controlada la arritmia orienta hacia el diagnóstico de $\mathrm{MCI}^{6,7}$. El diagnóstico correcto sólo puede establecerse después de demostrar la mejoría de la función del VI dentro de algunas semanas o meses después del tratamiento de la arritmia ${ }^{7,8}$.

En el flúter auricular es necesario con frecuencia controlar el ritmo y la cardioversión eléctrica es un método efectivo; dado el riesgo de recurrencia, se sugiere la ablación por catéter como tratamiento de elección, con una tasa elevada de éxito y baja de complicaciones en el flúter auricular típico, que además evita la toxicidad por fármacos ${ }^{2}$ (esta paciente cursó con arritmia ventricular por prolongación adquirida del QT). Tras la ablación del flúter auricular en pacientes con DSVI concomitante se advierte una mejoría de la función del VI en más del $50 \%$ de pacientes, y la normalización completa en casi el $75 \%$ de estos casos ${ }^{4}$.

Si bien el tratamiento decisivo es el control de la arritmia, los fármacos modificadores de la enfermedad (inhibidor de la enzima convertidora de angiotensina, betabloqueadores, antagonistas de receptores de mineralocorticoides) desempeñan un papel importante en la reversión del remodelamiento 2,7 .

\section{Conclusión}

Las arritmias son una causa poco reconocida de disfunción sistólica del VI. Si bien la FA es la forma más frecuente de arritmias causantes de MCIA, el flúter auricular es otra de sus causas principales. La recuperación clínica y ecocardiográfica después del control de la arritmia confirma el diagnóstico. La ablación por catéter es la mejor opción terapéutica, con mejoría de síntomas y recuperación de la función sistólica del VI total o parcial; en virtud de su reversibilidad, el tratamiento debe instituirse en un momento temprano. Existe riesgo de recurrencia y son necesarios un seguimiento muy cercano y el tratamiento radical en caso de arritmias recurrentes.

\section{Financiamiento}

Esta investigación no ha recibido ninguna beca específica de agencias de los sectores público, comercial o sin ánimo de lucro.

\section{Conflicto de intereses}

Ninguno.

\section{Responsabilidades éticas}

Protección de personas y animales. Los autores declaran que para esta investigación no se han realizado experimentos en seres humanos ni en animales.

Confidencialidad de los datos. Los autores declaran que han seguido los protocolos de su centro de trabajo sobre la publicación de datos de pacientes.

Derecho a la privacidad y consentimiento informado. Los autores han obtenido el consentimiento informado de los pacientes o sujetos referidos en el artículo. Este documento obra en poder del autor de correspondencia.

\section{Bibliografía}

1. Sugumar $H$, Prabhu S, Voskoboinik A, Peter M. Arrhythmia induced cardiomyopathy. J Arrhythmia. 2018;34:376-383.

2. Reyes-Caorsi W, Varela G, Tortajada G, Cortellezzi Z. Miocardiopatía inducida por arritmias. Revisión breve. Rev Urug Cardiol. 2019;34:122129 doi: $10.29277 /$ cardio.34.1.21

3. Velázquez-Rodríguez E, Martínez-Enríquez A. Cardiomiopatía dilatada inducida por taquicardia auricular ectópica. Arch Inst Cardiol Méx. 2000;70:292-300.

4. Brembilla-Perrot B, Ferreira JP, Manenti V. Predictors and prognostic significance of tachycardiomyopathy: insights from a cohort of 1269 patients undergoing atrial flúter ablation. Eur J Heart Fail. 2016;18:394-401.

5. Donghua Z, Jian P, Zhongbo X. Reversal of cardiomyopathy in patiens with congestive heart failure secondary to tachycardia. J Interv Card Electrophysiol. 2013;36:27-32.

6. Huízar JF, Ellenbogen KA, Tan AY, Kaszala K. Arrhythmia-induced cardiomiopathy. JACC-state-of-the-art review. J Am Coll Cardiol. 2019;73(18)

7. Ulus T, Okyay K, Kabul HK, Özcan EE, Özeke Ö, Altay H. Turkish Society of Cardiology consensus paper on management of arrhythmia-induced cardiomyopathy. Anatol J Cardiol 2019;21:98-106.

8. Serra JL, Figueroa JA, Fassano N. Taquicardia auricular focal y taquimiocardiopatía. Rev Fed Arg Cardiol. 2018;47(1):03-09. 